\title{
Ni/Photoredox-Catalyzed Enantioselective Cross-Electrophile Cou- pling of Styrene Oxides with Aryl Iodides
}

\author{
Sii Hong Lau ${ }^{\dagger}$ Meredith A. Borden,${ }^{\dagger}$ Talia J. Steiman, ${ }^{\star}$ Lucy S. Wang, ${ }^{\star}$ Marvin Parasram, and Abigail \\ G. Doyle* \\ Department of Chemistry, Princeton University, Princeton, New Jersey 08544, United States
}

Supporting Information Placeholder

\begin{abstract}
A Ni/photoredox-catalyzed enantioselective reductive coupling of styrene oxides and aryl iodides is reported. This reaction affords access to enantioenriched 2,2-diarylalcohols from racemic epoxides via a stereoconvergent mechanism. Multivariate linear regression (MVLR) analysis with 29 bioxazoline $(\mathrm{BiOx})$ and biimidazoline $(\mathrm{BiIm})$ ligands revealed that enantioselectivity correlates with electronic properties of the ligands, with more electron-donating ligands affording higher ee's. Mechanistic studies were conducted, lending support to the hypothesis that the electronic character of the ligands influences the enantioselectivity by altering the position of the transition state structure along the reaction coordinate. This study demonstrates the benefits of utilizing statistical modeling as a platform for mechanistic understanding and provides new insight into an emerging class of chiral ligands for stereoconvergent $\mathrm{Ni}$ and $\mathrm{Ni} /$ photoredox cross-coupling.
\end{abstract}

Epoxides are among the most versatile building blocks in organic synthesis due to their availability from olefins and proclivity toward ring-opening by various nucleophiles. ${ }^{1}$ Moreover, advances in asymmetric catalytic epoxidation have made enantiomerically-enriched epoxides useful chiral precursors for stereospecific ring-opening. ${ }^{2}$ Alternatively, chiral catalyst-controlled asymmetric ring-opening of epoxides represents an attractive method for enantioselective synthesis (Figure 1A). ${ }^{3}$ Significant and enabling advances in this area have been realized predominantly with soft or heteroatom-centered nucleophiles, such as azide, water, and cyanide. ${ }^{4}$ While asymmetric catalytic $\mathrm{C}-\mathrm{C}$ bond formation can also be achieved using organolithium and organomagnesium reagents, these methods suffer from harsh conditions and poor functional group tolerance. ${ }^{5}$ Furthermore, reactions with chiral epoxide substrates proceed by kinetic resolution owing to a stereospecific ring-opening step. For both practical and fundamental reasons, development of chiral catalyst-controlled stereoconvergent $\mathrm{C}-\mathrm{C}$ bond-forming reactions of racemic epoxides would be of high value.

Transition-metal catalyzed cross-coupling offers a mild and versatile approach to $\mathrm{C}-\mathrm{C}$ bond formation with the potential to effect chiral catalyst control. ${ }^{6}$ Over the past two decades, our group, alongside the groups of Jamison and Weix, have described strategies to engage epoxides as electrophiles in Ni-catalyzed cross-coupling. ${ }^{7}$ Weix and coworkers reported the first enantioselective cross-electrophile coupling with meso-epoxides using a chiral titanocene cocatalyst in conjunction with a racemic Ni catalyst (Figure 1B). ${ }^{8}$ More recently, the Yamamoto group described the arylation of 3,4-epoxyalcohols using chiral bioxazoline (BiOx) ligands and $\mathrm{Ni}$ catalysis that furnishes cross-coupled products in excellent enantio- and diastereoselectivity (Figure 1B). ${ }^{9}$ In this reaction, a pendant alcohol directing group is required on the epoxides for high stereoinduction. These important advances notwithstanding, the discovery and development of complementary methods, particularly to address the challenge of stereoconvergent cross-coupling with racemic terminal epoxides, is necessary to expand the scope and generality of this approach.

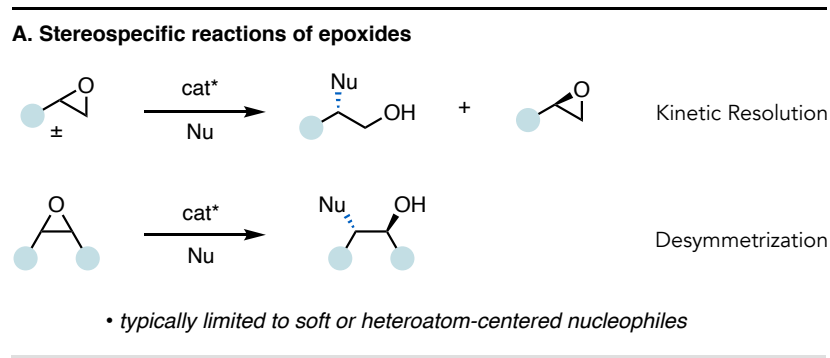

B. Prior work: asymmetric Ni-catalyzed reductive coupling with epoxides

Weix:
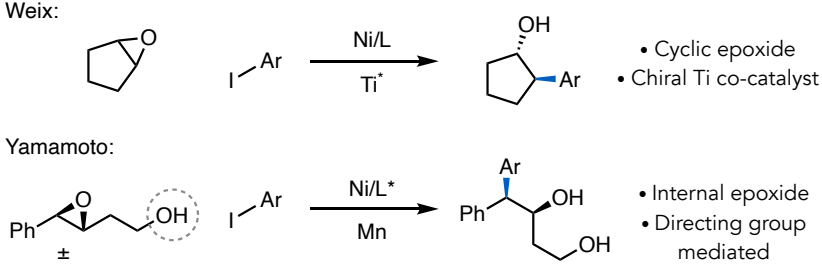

C. This work: undirected stereoconvergent coupling with terminal epoxides

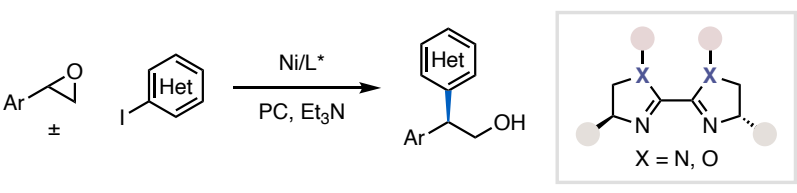

- regio- and enantioselective $\quad \cdot$ mild conditions $\quad \cdot$ mechanistic insights from MVLR

Figure 1. Strategies for asymmetric synthesis with epoxides. 
Recently, our group reported a photo-assisted reductive coupling (PARC) of racemic epoxides with aryl iodides via the merger of Ni-, Ti-, and photoredox catalysis. ${ }^{7 \mathrm{e}}$ Mechanistic studies revealed that $\mathrm{C}-\mathrm{C}$ bond formation with styrene oxides proceeds in a stereoablative manner, suggesting that the development of a stereoconvergent coupling of racemic epoxides with a chiral catalyst was mechanistically feasible. Herein, we describe a $\mathrm{Ni} /$ photoredox-catalyzed enantioselective crosselectrophile coupling of styrene oxides with aryl iodides using a chiral biimidazoline (BiIm) ligand (Figure 1C). This transformation allows facile access to enantioenriched 2,2-diarylalcohols, which are privileged motifs in bioactive molecules. ${ }^{10}$ Whereas steric effects are typically responsible for the origin of stereoselectivity, multivariate linear regression (MVLR) analysis with $\mathrm{BiOx}$ and $\mathrm{BiIm}$ ligands revealed that the electronic character of the ligands is the main contributor to enantioinduction. Further experimental studies were conducted to interrogate this statistical model, ultimately providing support for a nonintuitive structure-selectivity relationship that may be of use in the design of other enantioselective $\mathrm{Ni}$ /photoredox cross-coupling reactions.

Our optimization efforts focused on identifying an appropriate chiral ligand for the coupling of styrene oxide $\mathbf{1}$ and aryl iodide 2 using conditions derived from our prior work in racemic PARC of epoxides. ${ }^{7 e, 11}$ Initial evaluation of common chiral amine-based bidentate ligands indicated that a variety of $\mathrm{BiOx}$ ligands-a high performing ligand class in our prior report on asymmetric reductive coupling of aziridines-offered good levels of enantioinduction (Figure S1, S2). ${ }^{12}$ However, the desired cross-coupled product $\mathbf{3}$ was formed in low to moderate yield. Further optimization of other reaction components using $\mathbf{L} \mathbf{1}$ as the ligand revealed that the titanocene cocatalyst required in our previously reported method is not needed in this transformation, while addition of catalytic $\mathrm{MgCl}_{2}$ as a salt additive increases the reaction yield (Table 1 , entry $1-3) .{ }^{13}$

Recently, chiral biimidazoline (BiIm) ligands ${ }^{14}$ were shown to be effective in several enantioselective Ni-catalyzed reactions such as a benzylic $\mathrm{C}-\mathrm{H}$ arylation and hydroarylation of vinylarenes. ${ }^{15}$ Although the ligand class has not been applied to asymmetric cross-electrophile coupling, the structural similarity of BiIm and $\mathrm{BiOx}$ ligands, coupled with their additional site for derivatization, drew our interest. We prepared a small selection of known BiIm ligands (L4-L6) and evaluated them on our model reaction (entry 6-8). BiIm ligand L6 furnished the desired product in $66 \%$ yield and $89 \%$ ee. Performing the reaction in a photoreactor further improved the yield to $70 \%$ with a slight increase in enantioselectivity to $91 \%$ ee (entry 9 ). ${ }^{16}$ Control experiments indicated that $\mathrm{Ni}$, ligand, photocatalyst, ${ }^{17}$ triethylamine, and light are required for the transformation (entry 10, 11).

Table 1. Reaction optimization.

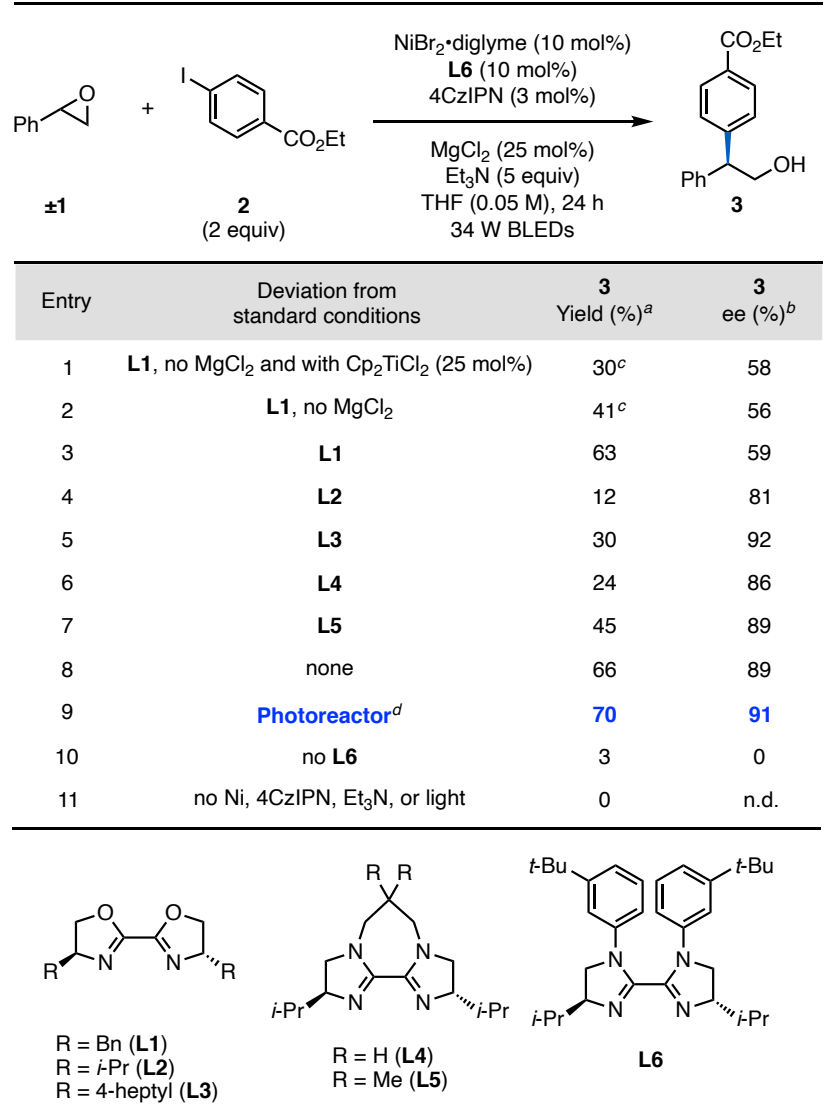

${ }^{a}$ Determined by GC $(0.05 \mathrm{mmol}) .{ }^{b}$ Determined by chiral HPLC. ${ }^{c}$ Determined by NMR. ${ }^{d}$ Penn $\mathrm{PhD}$ photoreactor $(450 \mathrm{~nm})$ on 0.5 mmol scale.

With the optimized reaction conditions, we examined the reaction scope with respect to aryl iodides (Table 2). A wide range of para-substituted electron-rich and electron-deficient aryl iodides (3-9) underwent coupling in high enantioselectivity. In general, more electron-deficient aryl iodides afforded higher yields than electron-rich substrates. The reaction tolerates aryl iodides containing chlorine (5), pinacol boronic ester (9), and protic acetamide (8) groups, all of which may serve as functional group handles for further diversification. Nitrogen- and oxygen-containing heterocycles such as pyridine and 2,3-dihydrobenzofuran $(\mathbf{1 4}, \mathbf{1 5})$ are also well tolerated, demonstrating the potential for this protocol to be used in the synthesis of bioactive compounds. Additionally, while meta-substituted aryl iodides (10-12) are competent substrates under the reaction conditions, ortho-substituted aryl iodides delivered trace product. As evidence of the stereoconvergent nature of the reaction, subjecting both $R$ and $S$ enantiomers of styrene oxide 1 to the standard reaction conditions generated the enantioenriched product 3 in $63 / 75 \%$ yield and $91 / 91 \%$ ee respectively. ${ }^{18}$ 
Table 2. Scope of aryl iodide.

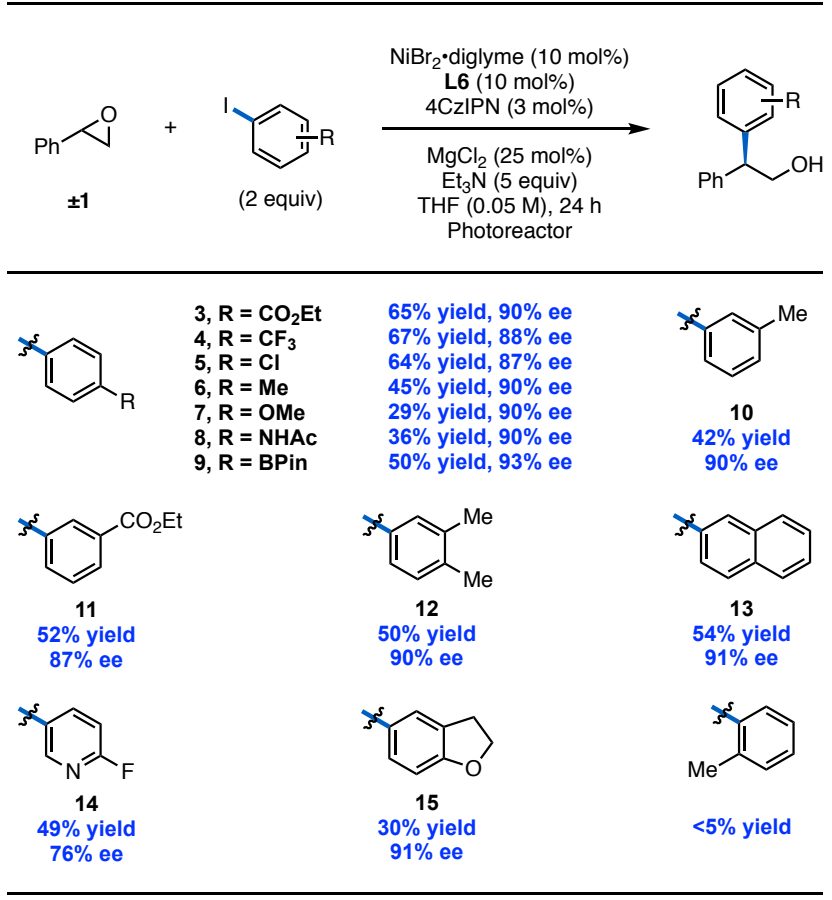

${ }^{a}$ Yield and ee are average of two runs $(0.5 \mathrm{mmol})$.

Next, we proceeded to examine the scope of styrene oxides (Table 3). A wide range of meta- and para-substituted styrene oxides with electron-donating and electron-withdrawing functionalities (16-22) were compatible under the reaction conditions, generating products in moderate to good yield and ee's. Sterically hindered 2(o-tolyl)oxirane (23) underwent cross-coupling, albeit in lower yield and enantioselectivity. Nevertheless, the compatibility of ortho-substitution on the styrene oxide offers a strategic alternative to the limitation in the aryl iodide scope.

Table 3. Scope of epoxides.

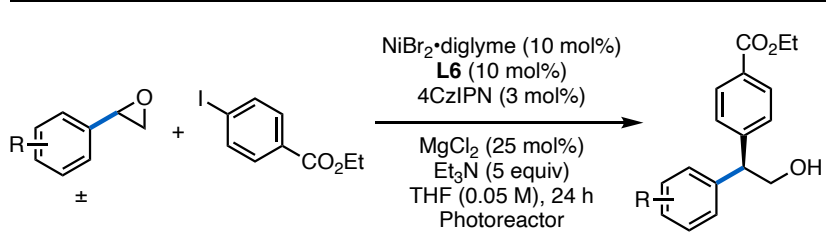

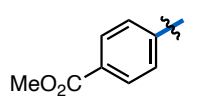

16

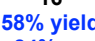

$84 \%$ ee

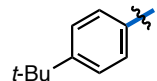

20

$70 \%$ yield

$89 \%$ ee

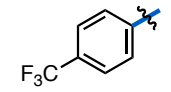

17

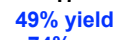

$74 \%$ ee

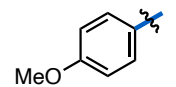

21

$31 \%$ yield

$89 \%$ ee

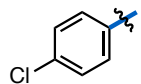

18

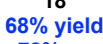

$78 \%$ ee

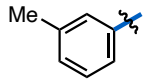

22

$63 \%$ yield $85 \%$ ee

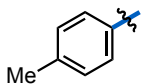

19 $51 \%$ yield $88 \%$ ee

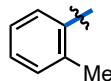

23 $16 \%$ yield
${ }^{a}$ Yield and ee are average of two runs $(0.5 \mathrm{mmol})$.

In a prior report from our group on Ni-catalyzed enantioselective reductive coupling of aziridines, we performed MVLR analysis in collaboration with the Sigman lab using $17 \mathrm{BiOx}$ ligands. ${ }^{12}$ Since a 1,1-diarylmethane stereocenter is generated in both the epoxide and aziridine coupling reactions, we questioned whether similar effects might apply in the current study. We therefore sought to use statistical and computational tools to understand the key structural features of the BiIm ligands that influence enantioselectivity in the epoxide coupling, and through accompanying mechanistic studies, shed light on aspects of the reaction mechanism that are otherwise difficult to evaluate. To do so, we gathered enantioselectivity data from an extended scope of $\mathrm{BiOx}$ and BiIm ligands, generated computationally-derived features of the ligands, and performed MVLR analysis. ${ }^{19}$

A total of $20 \mathrm{BiOx}$ and $9 \mathrm{BiIm}$ ligands with diverse structure were evaluated under the reaction conditions shown in Figure 2A. Ground state structures were calculated by DFT at the M06$2 \mathrm{X} /$ def2TZVP ${ }^{20}$ level of theory for three different coordination states of the ligands: the free ligand which was used in the aziridine study, a tetrahedral $\mathrm{L} * \mathrm{NiF}_{2}$ complex that serves as the most cost-effective surrogate to restrict the flexibility of the ligand, and a square planar $\mathrm{L} * \mathrm{Ni}(p$-tolyl $) \mathrm{Cl}$ complex which resembles possible on-cycle species in the catalytic cycle (Figure $2 \mathrm{~B}$ ). ${ }^{21}$ Subsequently, features were acquired from these structures and were related to the enantioselectivity (expressed as $\Delta \Delta \mathrm{G}^{\ddagger}$ ) in the MVLR analysis. By comparing models built from molecular descriptors extracted from different representations of the ligand, we sought to probe the structural complexity and associated computational cost of ligand representation sufficient to create a statistically robust descriptive model.

To assess predictive ability of a statistical model, leave-oneout and leave- $p$-out cross-validation are commonly used, especially in the context of small datasets. However, such methods may yield seemingly good performance metrics as an overwhelming majority of the dataset is used to train the model. ${ }^{22}$ Instead, we employed a repeated stratified nested cross-validation method consisting of two cross-validation loops wherein the data was divided into train-validation/test splits and the inner loop is used to select regressor features (Figure 2B). This method has been shown to provide an almost unbiased estimate of true performance error in the identification of a robust predictive model. ${ }^{23}$

The best-performing model for each ligand representation was selected based on the number of times that model appeared to rank the highest (performance evaluated by RMSE) among the outer folds (Figure $2 \mathrm{C}$ ). For $\mathrm{L}^{*} \mathrm{Ni}(p$-tolyl)Cl complex, the final linear regression model $\left(\operatorname{adj} . \mathrm{R}^{2}=0.74\right)$ consists of three independent parameters: $\boldsymbol{N B O}_{N 1}$ (average NBO charge of the oxazoline/imidazoline nitrogen atoms), $\boldsymbol{N B O}_{\boldsymbol{C} 4}$ (average NBO charge of carbon atoms adjacent to the oxazoline/imidazoline ring), and $\boldsymbol{P o l}$ (polarizability). Similarly, the three-parameter models for the $\mathrm{L} * \mathrm{NiF}_{2}$ and free ligand representations exhibit at least two electronic parameters, highlighting the importance of ligand electronic character on the enantioselectivity. However, these models performed worse, giving adj. $\mathrm{R}^{2}$ of 0.69 and 0.68 respectively. We also subjected each model to a $5 \times 2$ cross-validation test to compare model performance across different coordination states (See SI). ${ }^{24}$ Overall, while more precise descriptors can be obtained from $\mathrm{L}^{*} \mathrm{Ni}(p$-tolyl $) \mathrm{Cl}$, our studies indicate that the free ligand descriptors are sufficient in constructing a descriptive model, thereby saving computational cost. 


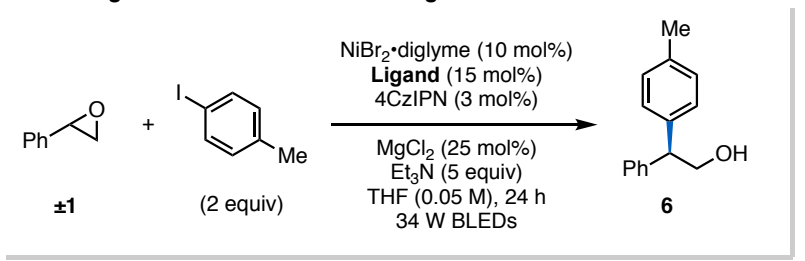

Representative examples (from $20 \mathrm{BiOx}$ and 9 Bilm):

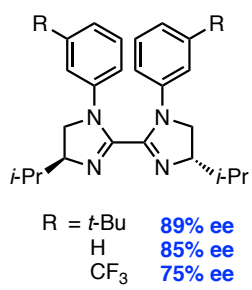

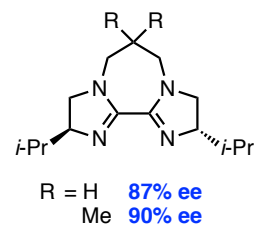
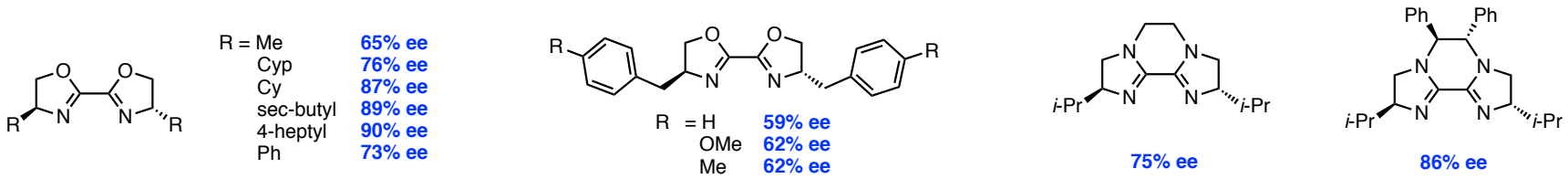

B. Comparison of ligand- and complex-based models using nested cross-validation

Nested cross-validation scheme:

outer folds: performance assessment

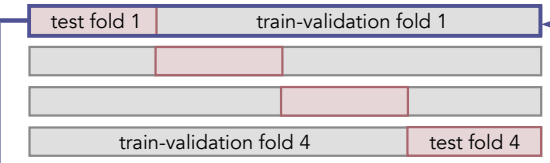

inner folds: feature selection

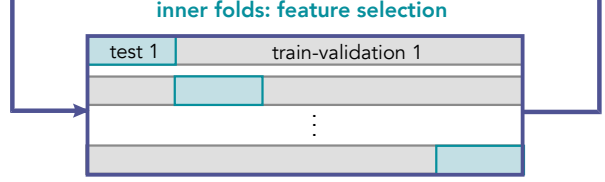

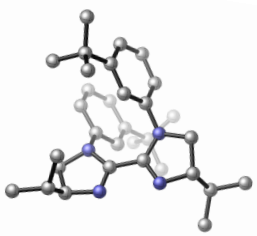

free ligand
$\Delta \Delta \mathrm{G}^{\ddagger} \sim \mathrm{NBO}_{\mathrm{C} 2}+\mathrm{NBO}_{\mathrm{C} 4}+\mathrm{Pol}$

Adj. $R^{2}=0.68$ Count (out of 20 folds) $=\mathbf{5}$

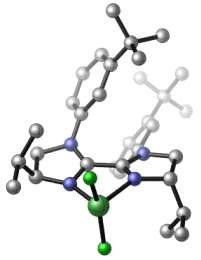

$\mathrm{L}^{\star} \mathrm{NiF}_{2}$ complex $\Delta \Delta \mathrm{G}^{\ddagger} \sim \mathrm{HOMO}+\mathrm{NBO}_{\mathrm{C} 4}+v_{\mathrm{CNsa}}$

Adj. $R^{2}=0.69$ Count (out of 20 folds) $=\mathbf{5}$

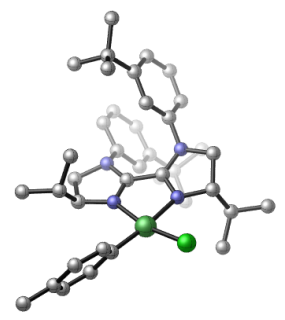

L*Ni(p-tolyl)Cl complex $\Delta \Delta \mathrm{G}^{\ddagger} \sim \mathrm{NBO}_{\mathrm{N} 1}+\mathrm{NBO}_{\mathrm{C} 4}+\mathrm{Po}$

Adj. $R^{2}=\mathbf{0 . 7 4}$ Count (out of 20 folds) $=12$

\section{Evaluation of ligand features in MVLR and via PCA analysis}

For L*Ni(p-tolyl)Cl:

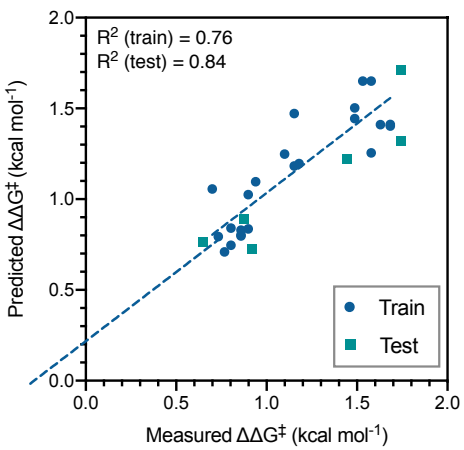

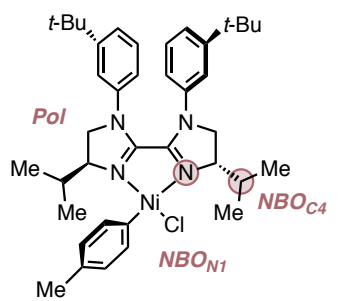

$\Delta \Delta \mathrm{G}^{\ddagger}=-0.58 N B O_{N 1}+0.46 N B O_{C 4}-0.27 \mathrm{Pol}$

enantioselectivity governed by electronic effects
BiOx and Bilm separated by electronic effects (PC1); $\mathrm{BnBiOx} \&$ non-BnBiOx separated by polarizability (PC2)

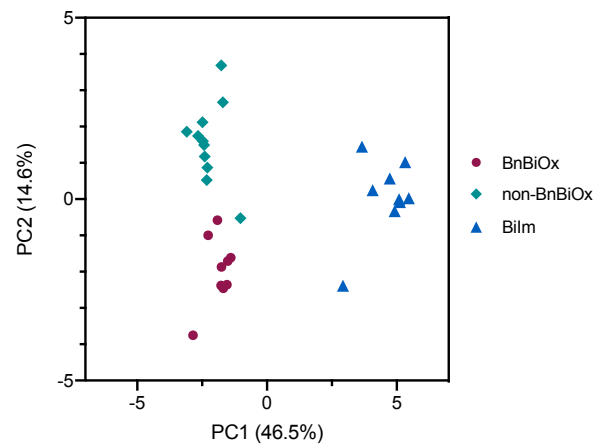

Figure 2. Computational and statistical analysis.

Since the models were acquired from scaled parameters, the magnitude and sign of the coefficients can give information about the effects of the features. For the $\mathrm{L}^{*} \mathrm{Ni}(p$-tolyl $) \mathrm{Cl}$ model, enantioselectivity is largely governed by electronic effects, with more electron-donating ligands delivering higher levels of enantioselectivity (Figure 2C, middle). On the other hand, enantioselectivity is negatively correlated to the polarizability. ${ }^{25}$ To better visualize the features, we performed dimensionality reduction using principal component analysis (PCA) and plotted the data to show clusters based on their similarity (Figure $2 \mathrm{C}$, right). We found that $\mathrm{BiOx}$ and BiIm ligands are separated by PC1 (46\%) whose loadings are highly weighted toward electronic features, whereas PC2 (14\%) splits BiOx into two clusters- $\mathrm{Bn}-\mathrm{BiOx}$ and non-BnBiOx -based mostly on polarizability and steric features. 


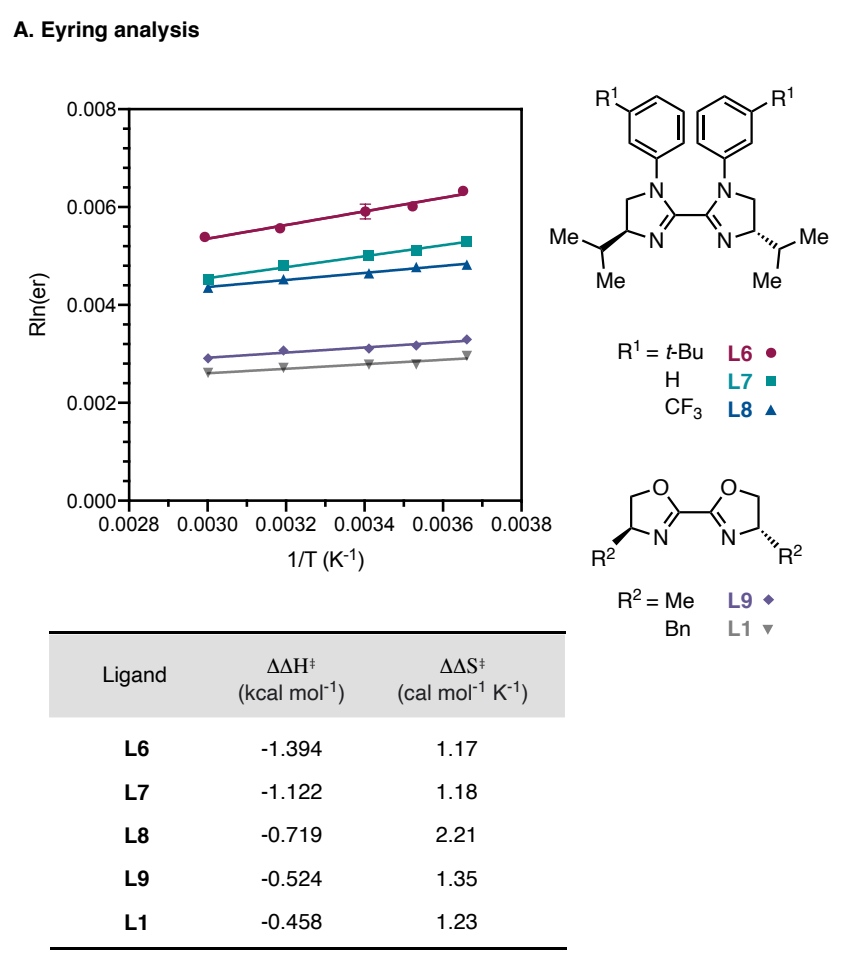

B. Correlation between $\mathrm{NBO}_{\mathrm{N} 1}$ and $\Delta \Delta \mathrm{H}^{\ddagger}$

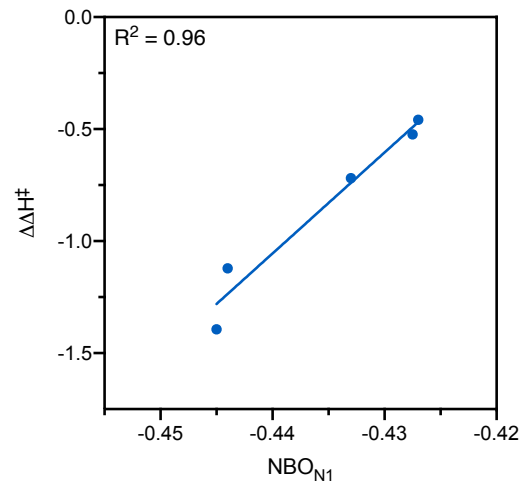

C. Ligand electronic character affects position of transition state

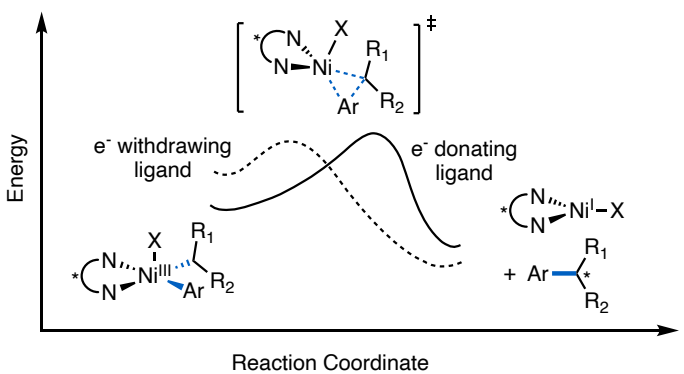

Figure 3. Mechanistic Studies.

Finally, we sought to investigate how the electronic character of the ligands might influence enantioselectivity and experimentally validate the model. A seminal report by Jacobsen and coworkers demonstrated that more electron-donating Mn-salen catalysts led to higher enantioselectivity in an asymmetric epoxidation reaction as a result of a later, more product-like transition state in accordance with Hammond's postulate. ${ }^{26}$ This prompted us to examine if a similar phenomenon was occurring in our system. An Eyring analysis was performed to determine the $\Delta \Delta \mathrm{H}^{\ddagger}$ and $\Delta \Delta \mathrm{S}^{\ddagger}$ between the major and minor diastereomeric transition states leading to both enantiomeric products. Energetic parameters were obtained on the model reaction using a systematic series of electronically distinct BiIm (L6-L8) and $\mathrm{BiOx}$ ligands $(\mathbf{L 9}, \mathbf{L 1})$ from 0 to $60^{\circ} \mathrm{C}$. We found that the enthalpic component $\left(\Delta \Delta \mathrm{H}^{*}\right)$ of these reactions exhibits an upward trend with more electron-donating ligands, while the entropic contribution $\left(\Delta \Delta \mathrm{S}^{\ddagger}\right)$ does not show a clear trend (Figure $3 \mathrm{~A})$. In addition, the experimental $\Delta \Delta \mathrm{H}^{\ddagger}$ is highly correlated with the calculated molecular charge feature $\boldsymbol{N B} \boldsymbol{O}_{\boldsymbol{N} 1}\left(\mathrm{R}^{2}=\right.$ 0.96), suggesting that more selective ligands within this study rely on enthalpic factors to reach a later, product-like transition state (Figure 3B).

Prior computational studies of related coupling reactions have suggested that either reductive elimination from $\mathrm{Ni}$ (III) or radical addition to tetrahedral $\mathrm{Ni}(\mathrm{II})$ is the enantiodetermining step. $^{27,28}$ In terms of substrate and ligand identity, this present work is more analogous to the system studied by Gutierrez, Kozlowski, and Molander where reductive elimination is enantiodetermining. In this case, a more electron-donating ligand would be expected to better stabilize $\mathrm{Ni}$ (III) leading to a less exergonic step (Figure 3C). However, our results do not rule out the possibility that radical addition is stereodetermining. Further experimental and computational studies are necessary to interrogate these possibilities.

In conclusion, we have developed a $\mathrm{Ni}$ /photoredox catalyzed stereoconvergent coupling of styrene oxides with aryl iodides. Our study highlights the use of statistical modeling to elucidate a structure-selectivity relationship within a class of catalytic reactions that are otherwise mechanistically quite complex. These mechanistic findings offer insight into the design of improved chiral ligands in stereoconvergent $\mathrm{Ni}$ and $\mathrm{Ni}$ /photoredox crosscoupling.

\section{ASSOCIATED CONTENT}

\section{Supporting Information}

The Supporting Information is available free of charge on the ACS Publications website.

Experimental details, optimization studies and characterization data (PDF)

\section{AUTHOR INFORMATION}

\section{Corresponding Author}

Abigail G. Doyle - Department of Chemistry, Princeton University, Princeton, New Jersey 08544, United States; orcid.org/0000-0002-6641-0833; Email: agdoyle@ princeton.edu

\section{Authors}

Sii Hong Lau - Department of Chemistry, Princeton University, Princeton, New Jersey 08544, United States; orcid.org/0000-0002-2178-0187

Meredith A. Borden - Department of Chemistry, Princeton University, Princeton, New Jersey 08544, United States; orcid.org/0000-0001-5369-8199

Talia J. Steiman - Department of Chemistry, Princeton University, Princeton, New Jersey 08544, United States 
Lucy S. Wang - Department of Chemistry, Princeton University, Princeton, New Jersey 08544, United States

Marvin Parasram - Department of Chemistry, Princeton University, Princeton, New Jersey 08544, United States; orcid.org/0000-0002-6052-0417

\section{Author Contributions}

These authors contributed equally.

*These authors contributed equally.

\section{Funding}

The authors declare no competing financial interest.

\section{ACKNOWLEDGMENT}

Financial support was provided by NIGMS R35 GM126986, F32 Ruth L. Kirschstein NRSA Fellowship under Award No. F32 EB027587-01 (T.J.S) and F32 Ruth L. Kirschstein NRSA Fellowship under Award No. F32 GM129910-01 (M.P.). The authors gratefully acknowledge Dr. Andrzej Żurański for assistance in ligand parameterization and modeling discussions.

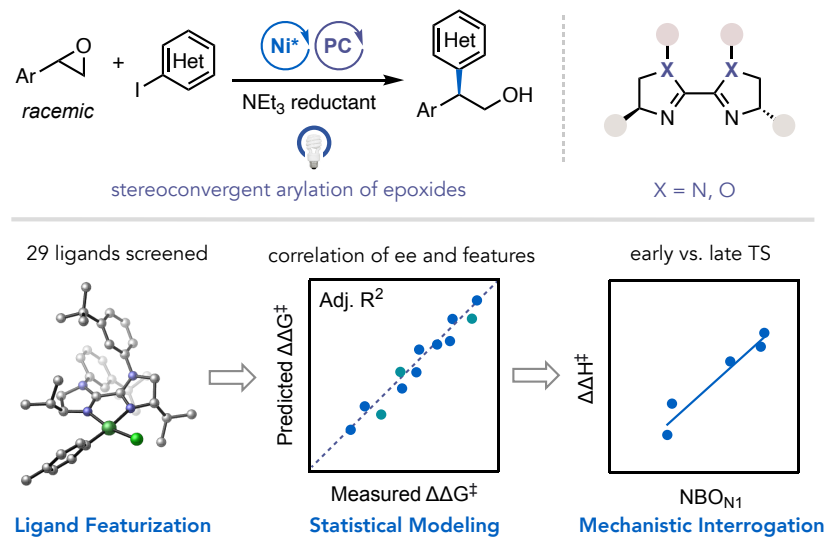




\section{REFERENCES}

(1) Smith, J. G. Synthetically Useful Reactions of Epoxides. Synthesis 1984, 1984, 629-656.

(2) For reviews on asymmetric epoxidation of olefins, see: (a) Johnson, R. A.; Sharpless, K. B. Asymmetric Oxidations and Related Reactions: Catalytic Asymmetric Epoxidation of Allylic Alcohols. In Catalytic Asymmetric Synthesis; John Wiley \& Sons, Ltd, 2000; pp 229-280. (b) Katsuki, T. Asymmetric Oxidations and Related Reactions: Asymmetric Epoxidation of Unfunctionalized Olefins and Related Reactions. In Catalytic Asymmetric Synthesis; John Wiley \& Sons, Ltd, 2000; pp $287-$ 325. (c) Zhu, Y.; Wang, Q.; Cornwall, R. G.; Shi, Y. Organocatalytic Asymmetric Epoxidation and Aziridination of Olefins and Their Synthetic Applications. Chem. Rev. 2014, 114, 81998256.

(3) Jacobsen, E. N. Asymmetric Catalysis of Epoxide RingOpening Reactions. Acc. Chem. Res. 2000, 33, 421-431.

(4) For reviews on asymmetric ring-opening of epoxides, see: (a) Lidskog, A.; Li , Y.; Wärnmark, K. Asymmetric Ring-Opening of Epoxides Catalyzed by Metal-Salen Complexes. Catalysts 2020, 10, 705. (b) Pastor, I.; Yus, M. Asymmetric Ring Opening of Epoxides. Curr. Org. Chem. 2005, 9, 1-29.

(5) Oguni, N.; Miyagi, Y.; Itoh, K. Highly Enantioselective Arylation of Symmetrical Epoxides with Phenyllithium Promoted by Chiral Schiff Bases and Salens. Tet. Lett. 1998, 39, 9023-9026.

(6) Huang, C.-Y.; Doyle, A. G. The Chemistry of Transition Metals with Three-Membered Ring Heterocycles. Chem. Rev. 2014, 114, 8153-8198.

(7) (a) Molinaro, C.; Jamison, T. F. Nickel-Catalyzed Reductive Coupling of Alkynes and Epoxides. J. Am. Chem. Soc. 2003, 125, 8076-8077. (b) Molinaro, C.; Jamison, T. F. Catalytic Reductive Coupling of Epoxides and Aldehydes: EpoxideRing Opening Precedes Carbonyl Reduction. Angew. Chem. Int. Ed. 2005, 44, 129-132. (c) Nielsen, D. K.; Doyle, A. G. Nickel-Catalyzed Cross-Coupling of Styrenyl Epoxides with Boronic Acids. Angew. Chem. Int. Ed. 2011, 50, 6056-6059. (d) Zhao, Y.; Weix, D. J. Nickel-Catalyzed Regiodivergent Opening of Epoxides with Aryl Halides: Co-Catalysis Controls Regioselectivity. J. Am. Chem. Soc. 2014, 136, 48-51. (e) Parasram, M.; Shields, B. J.; Ahmad, O.; Knauber, T.; Doyle, A. G. Regioselective Cross-Electrophile Coupling of Epoxides and (Hetero)Aryl Iodides via $\mathrm{Ni} / \mathrm{Ti} /$ Photoredox Catalysis. $A C S$ Catal. 2020, 10, 5821-5827.

(8) Zhao, Y.; Weix, D. J. Enantioselective Cross-Coupling of Meso-Epoxides with Aryl Halides. J. Am. Chem. Soc. 2015, 137, 3237-3240.

(9) Banerjee, A.; Yamamoto, H. Nickel Catalyzed Regio-, Diastereo-, and Enantioselective Cross-Coupling of 3,4-Epoxyalcohol with Aryl Iodides. Org. Lett. 2017, 19, 4363-4366.

(10) Ameen, D.; Snape, T. J. Chiral 1,1-Diaryl Compounds as Important Pharmacophores. MedChemComm 2013, 4, 893907.

(11) Initial reaction conditions: $\mathrm{NiBr}_{2} \cdot$ diglyme (10 mol\%), ligand (15 mol\%), 4CzIPN (5 mol\%), $\mathrm{Cp}_{2} \mathrm{TiCl}_{2}(25 \mathrm{~mol} \%$ or 0 mol\%), $\mathrm{Et}_{3} \mathrm{~N}$ (5 equiv), $\mathrm{MgCl}_{2}$ (25 mol\%), 2-(4-fluorophenyl)oxirane ( 1 equiv, $0.05 \mathrm{mmol})$, 4-iodobiphenyl (3 equiv), MeCN (0.033 M). See SI for more details.

(12) (a) Woods, B. P.; Orlandi, M.; Huang, C.-Y.; Sigman, M. S.; Doyle, A. G. Nickel-Catalyzed Enantioselective Reductive Cross-Coupling of Styrenyl Aziridines. J. Am. Chem. Soc. 2017, 139, 5688-5691. (b) Woods, B. P.; Orlandi, M.; Huang, C.-Y.; Sigman, M. S.; Doyle, A. G. Correction to "Nickel-Catalyzed Enantioselective Reductive Cross-Coupling of Styrenyl Aziridines." J. Am. Chem. Soc. 2018, 140, 7744-7745.

(13) $\mathrm{MgCl}_{2}$ is used as an additive to activate epoxides toward ring-opening. Magre, M.; Paffenholz, E.; Maity, B.; Cavallo, L.; Rueping, M. Regiodivergent Hydroborative Ring Opening of Epoxides via Selective C-O Bond Activation. J. Am. Chem. Soc. 2020, 142, 14286-14294.

(14) (a) Boland, N. A.; Casey, M.; Hynes, S. J.; Matthews, J. W.; Müller-Bunz, H.; Wilkes, P. Preparation of Enantiopure Biimidazoline Ligands and Their Use in Asymmetric Catalysis. Org. Biomol. Chem. 2004, 2, 1995-2002. (b) Hao, X.-Q.; Dong, Y.-N.; Gao, B.; Li, K.; Zhao, X.-M.; Xu, Y.; Song, M.-P. Biimidazoline Ligands for Palladium-Catalyzed Asymmetric Allylic Alkylation. Tetrahedron: Asymmetry 2015, 26, 1360-1368. (c) Li, J.; Yu, B.; Lu, Z. Chiral Imidazoline Ligands and Their Applications in Metal-Catalyzed Asymmetric Synthesis. Chin. J. Chem. 2021, 39, 488-514.

(15) (a) Cheng, X.; Lu, H.; Lu, Z. Enantioselective Benzylic C-H Arylation via Photoredox and Nickel Dual Catalysis. Nat. Commun. 2019, 10, 3549. (b) He, Y.; Liu, C.; Yu, L.; Zhu, S. Enantio- and Regioselective NiH-Catalyzed Reductive Hydroarylation of Vinylarenes with Aryl Iodides. Angew. Chem. Int. Ed. 2020, 59, 21530-21534. (c) Cuesta-Galisteo, S.; Schörgenhumer, J.; Wei, X.; Merino, E.; Nevado, C. NickelCatalyzed Asymmetric Synthesis of $\alpha$-Arylbenzamides. Angew. Chem. Int. Ed. 2021, 60, 1605-1609.

(16) Penn PhD photoreactor has been shown to provide better temperature control. Le, C. ; Wismer, M. K.; Shi, Z.-C.; Zhang, R.; Conway, D. V.; Li, G.; Vachal, P.; Davies, I. W.; MacMillan, D. W. C. A General Small-Scale Reactor To Enable Standardization and Acceleration of Photocatalytic Reactions. ACS Cent. Sci. 2017, 3, 647-653.

(17) The use of $\left.\operatorname{Ir}\left[\mathrm{dF}\left(\mathrm{CF}_{3}\right) \text { ppy }\right]_{2}(\mathrm{dtbbpy})\right) \mathrm{PF}_{6}$ in place of $4 \mathrm{CzIPN}$ delivered the product in similar yield and enantioselectivity, consistent with their similar excited state and ground state potentials. See SI. (a) Lowry, M. S.; Goldsmith, J. I.; Slinker, J. D.; Rohl, R.; Pascal, R. A.; Malliaras, G. G.; Bernhard, S. Single-Layer Electroluminescent Devices and Photoinduced Hydrogen Production from an Ionic Iridium(III) Complex. Chem. Mater. 2005, 17, 5712. (b) Luo, J.; Zhang, J. Donor-Acceptor Fluorophores for Visible-Light-Promoted Organic Synthesis: Photoredox/Ni Dual Catalytic $\mathrm{C}\left(\mathrm{sp}^{3}\right)-\mathrm{C}\left(\mathrm{sp}^{2}\right)$ Cross-Coupling. ACS Catal. 2016, 6, 873.

(18) Replacing $(\boldsymbol{S}, \boldsymbol{S})$-L6 with the opposite enantiomer $(\boldsymbol{R}, \boldsymbol{R})$ L6 under otherwise identical reaction conditions afforded the enantioenriched product $\mathbf{1 3}$ in comparable yield and opposite enantioselectivity of similar magnitude. See SI. 
(19) Sigman, M. S.; Harper, K. C.; Bess, E. N.; Milo, A. The Development of Multidimentsional Analysis Tools for Asymmetric Catalysis and Beyond. Acc. Chem. Res. 2016, 49, 12921301.

(20) This functional/basis set combination, previously used in our prior work on asymmetric aziridine coupling (ref. 12), was used for both free ligands and nickel complexes to ensure direct comparison among the different ligand representations. Model acquired using M06/def2TZVP level of theory provides comparable results. See SI for more details.

(21) Yin, H.; Fu, G. C. Mechanistic Investigation of Enantioconvergent Kumada Reactions of Racemic $\alpha$-Bromoketones Catalyzed by a Nickel/Bis(Oxazoline) Complex. J. Am. Chem. Soc. 2019, 141, 15433-15440.

(22) Hastie, T.; Tibshirani, R.; Friedman, J. The Elements of Statistical Learning: Data Mining, Inference, and Prediction.; Springer US: Boston, MA, 2009.

(23) Varma, S.; Simon, R. Bias in Error Estimation When Using Cross-Validation for Model Selection. BMC Bioinformatics 2006, 7, 91 .

(24) Dietterich, T. G. Approximate Statistical Tests for Comparing Supervised Classification Learning Algorithms. Neural Computation 1998, 10, 1895-1923.
(25) This observation can be attributed to the strong correlation between DFT-derived isotropic polarizability and the carbon count on the ligands, where the effect is more pronounced within BiOx ligands. See SI.

(26) (a) Jacobsen, E. N.; Zhang, W.; Güler, M. L. Electronic Tuning of Asymmetric Catalysts. J. Am. Chem. Soc. 1991, 113, 6703-6704. (b) Palucki, M.; Finney, N. S.; Pospisil, P. J.; Güler, M. L.; Ishida, T.; Jacobsen, E. N. The Mechanistic Basis for Electronic Effects on Enantioselectivity in the (Salen)Mn(III)Catalyzed Epoxidation Reaction. J. Am. Chem. Soc. 1998, 120, 948-954.

(27) (a) Gutierrez, O.; Tellis, J. C.; Primer, D. N.; Molander, G. A.; Kozlowski, M. C. Nickel-Catalyzed Cross-Coupling of Photoredox-Generated Radicals: Uncovering a General Manifold for Stereoconvergence in Nickel-Catalyzed Cross-Couplings. J. Am. Chem. Soc. 2015, 137, 4896-4899. (b) Yuan, M.; Song, Z.; Badir, S. O.; Molander, G. A.; Gutierrez, O. On the Nature of $\mathrm{C}\left(\mathrm{sp}^{3}\right)-\mathrm{C}\left(\mathrm{sp}^{2}\right)$ Bond Formation in Nickel-Catalyzed Tertiary Radical Cross-Couplings: A Case Study of Ni/Photoredox Catalytic Cross-Coupling of Alkyl Radicals and Aryl Halides. J. Am. Chem. Soc. 2020, 142, 7225-7234.

(28) Guo, L.; Yuan, M.; Zhang, Y.; Wang, F.; Zhu, S.; Gutierrez, O.; Chu, L. General Method for Enantioselective Three-Component Carboarylation of Alkenes Enabled by Visible-Light Dual Photoredox/Nickel Catalysis. J. Am. Chem. Soc. 2020, 142, 20390-20399. 